\title{
Programación de Rutas para Buses Urbanos en el Municipio de Fusagasugá a través de un Algoritmo de Aproximación
}

\author{
David Buriticá1, Reinaldo Sotoํㅜ, Oscar Perilla ${ }^{1}$
}

Recibido: Febrero 15 de 2014 Aprobado: Junio 20 de 2014

\begin{abstract}
Resumen:
El presente artículo utiliza un algoritmo de aproximación para organizar y normalizar los horarios de salida de buses urbanos. Se realizó una aplicación para el Sistema Integrado de Transporte de Fusagasugá, sobre un número finito de buses asignados a ciertas rutas. Se distribuyen la cantidad de viajes durante el día, estandarizando los horarios que deben cumplir bajo parámetros de demanda y capacidad para así prestar un servicio más adecuado.
\end{abstract}

Los resultados evidencian las bondades de la aplicación del algoritmo de aproximación propuesto en la programación de rutas en el transporte urbano.

Palabras Clave: Transporte, Logística, Algoritmos de aproximación, Programación, Transporte urbano.

\section{Abstract:}

This article uses an approximation algorithm to organize and standardize the departure times for urban buses. An application for Integrated Transport System of Fusagasugá was conducted through a representative sample, a finite number of buses assigned to certain routes. The number of trips is distributed throughout the day, standarizing schedules to be met within the parameters of demand and capacity, in order to provide a better service.

The results show the benefits of applying the algorithm and proposed approach in programming for urban transport routes

Key words: Transport, Logistic, Approximation algorithm, Scheduling, Urban transport.

\footnotetext{
1 Egresados del programa de Ingeniería Industrial de la Facultad de Ingeniería de la Corporación Universitaria Minuto de Dios - Sede Principal
} 


\section{INTRODUCCIÓN}

Un sistema de transporte urbano es un conjunto de elementos (infraestructuras, equipos, dispositivos de control el tránsito, elementos relacionados con el servicio que ofrece) que trabajan en forma integral con el objeto de suplir las necesidades de movilidad de personas [1].

Las grandes ciudades han visto la necesidad de implementar sistemas integrados de transporte del servicio público urbano, con el fin de reducir los problemas de movilidad que las aquejan. Sin embargo, se han presentado inconvenientes para la aplicación de dicho sistema, como por ejemplo el alto precio del combustible; el elevado consumo de tiempo en el trayecto, la accidentalidad y la contaminación ambiental [2].

El sistema de transporte público que predomina en Colombia es el Transporte Público Colectivo Urbano (TPCU) [1]. Este es accesible a todos, el usuario no se ocupa de otra cosa que de reservar el servicio y saber cuál es su destino. La empresa de transporte se encarga de todo lo referente a adquisición, mantenimiento, reposición, estacionamientos, etc. Son caracterizados por itinerarios fijos y recorridos según horarios [3]. En el municipio de Fusagasugá, Cundinamarca, se maneja este tipo de sistema, pero se percibe como un Sistema Integrado de Transporte ya que las tres empresas que operan de forma urbana - Cootransfusa, Tierra Grata y Expreso Fusacatán- se han unido con el fin de distribuir las rutas de forma equitativa.

Las empresas de transporte, para establecer los tiempos de sus rutas se basan en pronósticos. Estos requieren la aplicación de una sucesión de algoritmos matemáticos. Las expresiones matemáticas se determinan a partir de modelos que ordenan variables. Estos modelos de transporte además pueden ser utilizados en la evaluación de problemas que se presenten a futuro [2] [4]. Sin embargo, la realidad es otra y aun se ve reflejado en el gremio el uso ineficiente de personal no calificado que ordena las rutas según variables que no tienen en cuenta los factores clave: las rutas se determinan por la cantidad de buses y por criterios que, en algunos casos, no tienen bases metódicas sino empíricas [2].

En el sector de transporte de pasajeros o de carga, los tiempos que duran los buses, camiones, aviones o barcos en realizar sus viajes son vitales. Por lo tanto, un propósito de optimización es el de minimizar los tiempos de viaje, los cuales pueden ser mayores por el mal estado de las vías, el estado de los vehículos y otros factores asociados al tráfico vehicular [2]. En este artículo se propone un algoritmo de aproximación que se adapta al problema de programación de rutas urbanas. El algoritmo se fundamenta en la reducción del tiempo ocioso que se presenta en cada viaje programado. Por tanto, se establecen horarios para las salidas de buses con el fin de mejorar la frecuencia de las rutas, a fin de satisfacer la demanda del mercado, es decir los clientes. En el transporte público los tiempos de trayectos afectan los horarios de la programación de despachos que son vitales para la atención de usuarios. Si un bus se demora más de lo normal en realizar su ruta va a afectar al bus que va a salir detrás de este, ya que por demorarse más podrá recoger más pasajeros y por consiguiente afectará los ingresos del bus que venga detrás de él; esto se convierte en un efecto dominó, que afecta a toda la flota de buses de dicha ruta. [1]

El artículo muestra el desarrollo del algoritmo de aproximación que tiene como finalidad reducir el tiempo ocioso en la programación de rutas urbanas. Para ello, se realiza un análisis de los datos que arroja el algoritmo después de su aplicación, con el fin de dar a conocer la importancia de tener un orden de salida de los buses de diferentes rutas del Sistema Integrado de Transporte. Finalmente, se revisan los datos de salida del algoritmo para concluir sobre los resultados del mismo.

\section{ANTECEDENTES}

El problema de distribución de transporte, es abordado desde la Investigación de Operaciones, tal como se presenta por [9] y [10] a través de heurísticas (TSP y VRP entre otros) y Programación Lineal. En esta vía, se han realizado artículos que difieren en su aplicación, básicamente utilizan para su desarrollo Programación Lineal Entera [11] o heurísticas para resolución de problemas del transporte como los expuestos en [9]. El aporte del algoritmo de aproximación que se presenta consiste en determinar rutas urbanas a través de la reducción de tiempos ociosos.

Un algoritmo de aproximación se desarrolló en el año de 1950 por Toyoda y Ohno como parte de las técnicas de producción para el sistema de la compañía Toyota. Estas técnicas sirven para mejorar y optimizar los procesos operativos de cualquier compañía industrial, independientemente de su tamaño. Las técnicas de "Lean Manufacturing", como se les denomina, se utilizan en la optimización de las operaciones de 
forma que se puedan obtener tiempos de reacción más cortos, mejor atención, servicio al cliente, mejor calidad, costos más bajos, eliminación de cualquier actividad que no agregue valor al producto, servicio o proceso, eliminación de cualquier tipo de desperdicio (sobreproducción, retrasos, transporte, el proceso, inventarios, movimientos y calidad), mayor eficiencia del equipo, entre otros. [6] Todo esto se logra utilizando herramientas como los sistemas Kanban, el mantenimiento productivo total, los sistemas Kaizen, las 5's, Seis Sigma, Poka Yoke, Jidokas, entre otros que hacen parte del "Lean Manufacturing". Por lo tanto, el propósito fundamental con las técnicas de "Lean Manufacturing" es lograr que la empresa sea flexible para fabricar lo que el cliente quiere y en el momento en que lo pide, además de darle valor agregado a los productos [7] [8]. De acuerdo con lo anterior se desarrolló un algoritmo de aproximación basado en el concepto de "Lean Manufacturing" el cual busca integrar las cualidades descritas de este concepto y las técnicas empleadas en manufactura para generar un sistema de rutas urbanas en el municipio de Fusagasugá.

\section{METODOLOGÍA}

Para el desarrollo de la programación de rutas urbanas se realiza una analogía con el algoritmo de aproximación de manufactura esbelta propuesto por [5] y se propone la siguiente nomenclatura que se utiliza en el modelo de programación:

\begin{tabular}{|c|l|}
\hline \multicolumn{2}{|c|}{ Tabla 1. Variables } \\
\hline Símbolo & \multicolumn{1}{c|}{ Concepto } \\
\hline$i$ & Índice que representa las rutas tipo $\mathrm{i}$ \\
\hline$j$ & Índice que representa los buses tipo $\mathrm{j}$ \\
\hline$P j$ & Partes del componente $\mathrm{j}$ \\
\hline Capj & Capacidad del componente $\mathrm{j}$ \\
\hline Capmax & $\begin{array}{l}\text { Capacidad máxima que alcanza un } \\
\text { componente } \mathrm{j} \text { en el contexto }\end{array}$ \\
\hline$D p i$ & Demanda de personas total por ruta (día) \\
\hline$I j$ & Ingreso total diario por ruta \\
\hline$\$ P u$ & Precio unitario del pasaje. \\
\hline$K r i j$ & $\begin{array}{l}\text { Cantidad de tandas requeridas para } \\
\text { cumplir con la demanda }\end{array}$ \\
\hline$D r e a l$ & Demanda real que soporta el componente $\mathrm{j}$ \\
\hline$K i$ & $\begin{array}{l}\text { Capacidad promedio de carga de los buses } \\
\text { asignados a la ruta i. }\end{array}$ \\
\hline$F P i j$ & Frecuencia de salida del bus $\mathrm{j}$ en la ruta $\mathrm{i}$. \\
\hline$H S j$ & Tiempo de salida en minutos para el bus $\mathrm{j}$ \\
\hline$F P i j$ & Frecuencia de surtida de buses $\mathrm{j}$ \\
\hline
\end{tabular}

\begin{tabular}{|c|l|}
\hline Símbolo & \multicolumn{1}{|c|}{ Concepto } \\
\hline$t r$ & Tiempo de ruta \\
\hline$t d$ & Tiempo de descanso \\
\hline$n b$ & Numero de buses asignados a la ruta \\
\hline$H r$ & $\begin{array}{l}\text { Hora del día que marca el reloj en que } \\
\text { debe salir el bus j. }\end{array}$ \\
\hline$H S j$ & Tiempo de salida en minutos del bus j. \\
\hline$H j$ & Hora a la que empieza la jornada laboral. \\
\hline
\end{tabular}

Rutas (Tipos de Productos): las rutas son los recorridos dentro del municipio ya establecidas bajo ciertos parámetros de la empresa, indiferentes a este estudio.

Número de buses (Tamaño $Q^{*}$ ): los buses son el tamaño de "partes" necesarias para llevar a cabo el cumplimiento del servicio.

Demanda de Personas (Cantidad Requerida): la cantidad de personas que toman el servicio cada día en la ruta i. Para hallar este dato se tomaron los ingresos promedio de los conductores de cada ruta, debido a que la demanda de personas se restringe en cada ruta i por la capacidad de transporte del bus y se multiplicaron por la cantidad de buses asignados por la empresa a cada ruta. Luego ese valor se dividió por el precio unitario del pasaje:

$$
D p_{i}=\frac{\sum_{j=1}^{1} I_{j}}{\$ P u}, \text { Ecuación } 1.1
$$

Donde, $D p_{i}=$ demanda de personas total por ruta (cantidad por día)

$\mid j=$ Ingreso total diario por ruta

$\$ P u=$ Precio unitario del pasaje.

Empezar Jornada: Tiempo en el cual inicia la jornada laboral la ruta tipo i.

Componentes de la ruta (buses): los componentes de la ruta hace referencia a que requiere un número $\mathrm{Q}^{*}$ de buses que son necesarios para cumplir dicha ruta, es decir, son las "partes" con las que se realiza un "producto". Tiene valor uno (1), ya que un bus solo puede contarse una única vez, por ende este no toma un valor mayor a uno. Al lector se le plantea que se puede aplicar una heurística que tenga en cuenta el factor de capacidad (el modelo planteado 
asume que la capacidad de los buses es igual) y asigne horarios a los buses según su capacidad de carga con la siguiente ecuación:

$$
p_{j}=\frac{\operatorname{Cap}_{j}}{\operatorname{Cap}_{\text {max }}}, \quad \text { Ecuación } 1.2
$$

Donde,

$P_{j}$ : partes del componente j (relación \%)

Capj: capacidad del componente $\mathrm{j}$

Capmax: capacidad máxima que alcanza un componente $\mathrm{j}$ en el contexto.

Así pues la ecuación genera como resultado un grado de capacidad utilizada relacionando la capacidad real que tiene el vehículo y la capacidad máxima (disponible) que puede tener un vehículo en el municipio o ciudad. El algoritmo asignará a los buses de menor capacidad una frecuencia de salida más rápida y a los de mayor capacidad períodos más largos de espera para realizar sus recorridos.

Duración de la ruta (Tiempo de Surtida): la duración de la ruta es el tiempo que tarda un bus $j$ en recorrer la ruta $\mathrm{i}$ desde el punto de partida hasta volver al mismo sitio.

Capacidad del bus (Kanban): es la cantidad máxima de pasajeros que un bus puede transportar.

Descanso: el descanso es un tiempo entre rutas considerado dentro de las empresas de transporte como pausas activas, necesarias para mantener la condición productiva y psicológica del conductor.

\section{Datos de Entrada: preliminares}

El primer paso consiste en recolectar los datos referentes a las rutas y los buses que actualmente conforman el Sistema Integrado de Transporte de Fusagasugá. Se requirieron datos como el número de rutas del Sistema, el número de buses por cada ruta, la duración de cada ruta, la demanda de pasajeros y la cantidad de dinero que genera un bus en un viaje.

El número de pasajeros por minuto en cada ruta $\mathrm{N} / \mathrm{T}$.T se halla dividiendo la demanda diaria entre el tiempo diario total de cada ruta, por tanto:
Las siguientes etapas describen el desarrollo del algoritmo de aproximación para la programación de rutas:

\section{Etapa 1}

La Etapa 1 del algoritmo de aproximación de manufactura esbelta se basa en la conformación de lotes de producción con respecto a los productos tipo i. Para esta aplicación este modulo no tiene mayor significado, porque se parte del supuesto que el TSU (Tiempo de Surtida de Unidades) es el tiempo entre rutas, pero que los componentes (buses) tienen que realizar el recorrido y se entiende que análogamente el tiempo total de trabajo no está determinado por la suma de la cantidad de veces que dan la vuelta los buses, sino que al contrario esta dado por la capacidad máxima de trabajo diario por ruta, en otras palabras una conversión:

$$
T T T_{i}=H D_{i} * 60, \quad \text { Ecuación } 3.1
$$

Donde, TTT $_{i}$ : Tiempo total de trabajo diario por ruta (minutos)

$H D_{i}$ : horas del día que se trabaja por ruta

\section{Etapa 2}

Se toma el flujo diario de personas de la ruta i multiplicada por el factor de partes del componente $j$ (Ecuación 1.2) para hallar la demanda real que es capaz de soportar el bus j.

$$
D_{\text {real }}=p j * D p_{i}, \quad \text { Ecuación } 4.1
$$

Donde, $D_{\text {real: }}$ Demanda real que soporta el componente (cantidad de personas)

$\mathrm{Pj}$ : partes del componente $\mathrm{j}$

Dpi: demanda de personas total por ruta (día)

Así se compara con la capacidad promedio de los buses (el Kanban [5]) la cual permitirá establecer la cantidad de tandas o recorridos que se deben realizar en el día para suplir la demanda, con respecto a la capacidad del autobus. Esta fracción queda con unidades Personas ruta/Personas:

$$
K r_{i j}=\frac{D_{\text {real }}}{K_{i}}, \quad \text { Ecuación } 4.2
$$


Donde,

$K r_{i j}$ : Cantidad de tandas requeridas para cumplir

con la demanda

$D_{\text {real: }}$ : Demanda real que soporta el componente $\mathrm{j}$

$K_{i:}$ capacidad promedio de carga de los buses

asignados a la ruta $i$.

Luego se divide el tiempo total de trabajo diario por ruta (Tiempo/ruta) y la cantidad de tandas requeridas para cumplir con la demanda (Ruta) y hallar la frecuencia de salida de los buses.

$$
F P_{i j}=\frac{T T T_{i}}{\left(K r_{i j}\right)}, \quad \text { Ecuación } 4.3
$$

Donde,

FPij: frecuencia de salida del bus $\mathrm{j}$ en la ruta $\mathrm{i}$.

\section{Etapa 3}

Para lograr generar los horarios y el "scheduling" (programación) de salida de los buses se tienen en cuenta tres variables: duración de la ruta i, descanso asignado a la i ruta y la frecuencia de salida $\left(F P_{i j}\right)$.

La duración de la ruta i, $\left(t r_{i}\right)$ es una variable de tiempo y se determina externamente (variable exógena) por propiedades geográficas y demográficas de la ruta que la empresa ya ha evaluado. Por ende su valor es constante. El descanso asignado a la ruta $i$, también es una variable exógena que la empresa ha determinado $(t d)$.

\section{1) Raíz HS}

La raíz $\mathrm{HS}_{i}$ hace referencia a los tiempos de salida que se deben hacer desde el paradero para cada bus $j$, de la ruta $i$, en minutos. Para conocer el distanciamiento óptimo entre busetas tenemos la frecuencia de surtida de buses $F P_{i j}$. Cada bus tiene entonces una hora de salida, donde incluye el tiempo que dura la ruta más el descanso que tiene asignada cada ruta:

$$
H S j=\left\{\begin{array}{c}
0 s i j=1 \\
F P_{i j} * j s i 1<j \leq n b \\
t r+t d+H S_{j-n b} s i j>n b
\end{array}\right\}, \quad \text { Ecuación } 5.1
$$

Donde,

HSj: Tiempo de salida en minutos para el bus $\mathrm{j}$ tr: tiempo de ruta

$t d$ : tiempo de descanso

$n b$ : numero de buses asignados a la ruta

2) "Scheduling" de salida

Para generar el cronograma de salida se organizan todos los tiempos de salida asignados a cada bus $\mathrm{j}$ de la ruta i y se ordenan de menor a mayor. Esta parte del algoritmo es gráfico y se establece para llevar un control del orden en que van los buses $\mathrm{j}$ con respecto a los tiempos generados.

3) Horarios de Surtida

El resultado final del algoritmo es un cronograma que muestra el reloj al que el bus $j$, asignado a la ruta $i$, debe salir del paradero en cada recorrido que debe hacer en el día y la cantidad de recorridos que realiza. Para generar el reloj tenemos:

\section{Horas:}

$$
H r=\text { redondear }\left(\frac{H S_{j}}{60}+H j\right), \quad \text { Ecuación } 5.2
$$

Donde,

$H r:$ hora del día que marca el reloj en que debe salir el bus $j$.

HSj: tiempo de salida en minutos del bus $j$.

$H j$ : hora a la que empieza la jornada laboral.

La siguiente ecuación cumple la función de convertir la hora que marca el reloj (p.e. 5:20 am.) a una en horas $(\mathrm{Hj}=5,3$ a.m. $)$

\section{Minutos:}

$$
\begin{aligned}
& \frac{H S_{j}}{6}+H j-H r \\
& \quad \text { Min }=\quad)^{*} 60, \quad \text { Ecuación } 5.3
\end{aligned}
$$

Donde,

Min: minuto del día que marca el reloj en que debe salir el bus $\mathrm{j}$.

$H S j$ : tiempo de salida en minutos del bus $j$.

$H j$ : hora a la que empieza la jornada laboral.

$H r$ : hora del día que marca el reloj en que debe salir el bus $j$. 


\section{APLICACIÓN ALGORITMO DE APROXIMACIÓN}

\section{Programación}

La ejecución del algoritmo se hizo en Excel, el cual brinda una cantidad importante de recursos de fácil uso y acceso, asimismo permite un orden en el manejo de los datos.

\section{Datos de Entrada}

Para realizar el algoritmo es necesario contar con datos de entrada suficientes y así aplicar el modelo. Los datos fueron tomados del Sistema Integrado de Transporte de Fusagasugá. Allí tres empresas de transporte tienen un número " $n$ " de rutas $y$ son propietarias de un número " $m$ " de buses. Las rutas están definidas por códigos. Para delimitar el problema se tomaron tres rutas con la cantidad de buses que se le asignan a cada una de ellas. Las rutas elegidas fueron consideradas por su trascendencia, según los conductores.

\section{a) Maíz Amarillo}

Ruta que atraviesa el eje urbano de Fusagasugá, tiene como característica principal que rodea el centro y además provee el servicio de transporte a una de las zonas más alejadas y con una importante población: los barrios de interés social Ebenezer, Maíz Amarillo y Gran Colombia, los cuales quedan sobre uno de los costados de la vía Panamericana. Tiene asignada a ella un número de 19 buses diariamente.

\section{b) Pablo Bello Cedritos}

Ruta central que maneja la parte norte, centro y occidental de Fusagasugá. Es una de las rutas más importantes en el municipio, pues provee el servicio de transporte directo e indirecto a un número amplio de barrios de la ciudad. También es considerada por los conductores como una de las mejores en cuanto a rendimiento, ya que pasa cerca a varios de los colegios localizados en Fusagasugá, el centro y la zona de Pablo Bello y alrededores, las cuales son zonas residenciales. Tiene asignado diariamente un total de 19 buses.

\section{c) Llano Largo}

Ruta transversal al eje del municipio, mucho más amplia que Maíz Amarillo, abarca desde el barrio Llano Verde y Llano Largo hasta los límites del norte del municipio: La Clarita. Su recorrido confronta la zona comercial de Fusagasugá y es una de las rutas más largas en su recorrido.

Una vez establecidas estas dos variables, es importante conocer la demanda diaria del servicio por parte de la comunidad para cada ruta, para ello se tomó el dinero promedio producido por un conductor. Los conductores no tienen contadores que lleven sus cuentas, pero si tienen empíricamente el conocimiento de ellas, y muchos llevan libros donde registran sus cuentas año tras año. Para formular un supuesto que se aproxime a la realidad de la demanda diaria se tienen en cuenta la cantidad de buses asignados a la ruta, el promedio ponderado de todos y el precio del pasaje de la ciudad. Aplicando la ecuación 1.1 obtenemos la demanda por ruta de pasajeros (Tabla 2).

Los tiempos de duración de ruta y de descanso por ruta fueron establecidos por el consejo del Sistema Integrado de transporte. Los tiempos de duración de ruta son dados por las condiciones geográficas y del diseño de la ruta, por ende la duración de cada una y su longitud fueron determinadas como un ponderado del tiempo que tarda un bus en recorrer la trayectoria trazada. El descanso se argumenta por dos factores, la carga de trabajo diaria y la demanda de servicio de la ruta: los conductores en la mayoría de los casos trabajan más de las 12 horas y es por eso que se hace necesario que los conductores tengan tiempos de descanso entre cada vuelta dada, que en este caso está determinada con respecto a la duración del recorrido de cada ruta, entendido como una pausa activa en donde el conductor estaciona su bus y descansa. El segundo factor que determina el descanso, la demanda de servicio, hace referencia a que dependiendo de la cantidad de personas que demanda una ruta existe un descanso mayor o menor, que la empresa considera para organizar los tiempos de los buses (Tabla 3).

Tabla 2. Demanda de Personas por ruta, tabulación de número de buses por ruta y rutas elegidas. Fuente: Tabulación propia y suministrados por Hernán Buriticá Mejía conductor de Transportes Tierra Grata, empresa perteneciente al SITF.

\begin{tabular}{|c|c|c|c|c|c|c|c|}
\hline CÓD. & Ruta & T & $\begin{array}{c}\text { Producido por } \\
\text { día Unitario }\end{array}$ & $\begin{array}{c}\text { No. de } \\
\text { buses }\end{array}$ & $\begin{array}{c}\text { Producido total } \\
\text { por ruta }\end{array}$ & $\begin{array}{c}\text { Precio del } \\
\text { pasaje }\end{array}$ & Demanda \\
\hline 107 & Pablo Bello Cedritos & 56 & $\$ 300.000,00$ & 19 & $\$ 5.700 .000,00$ & $\$ 1.100,00$ & 5182 \\
\hline 103 & Maíz Amarillo & 53 & $\$ 240.000,00$ & 19 & $\$ 4.560 .000,00$ & $\$ 1.100,00$ & 4145 \\
\hline 100 & Llano Largo & 64 & $\$ 230.000,00$ & 20 & $\$ 4.600 .000,00$ & $\$ 1.100,00$ & 4182 \\
\hline
\end{tabular}


Tabla 3. Los tiempos de descanso y de duración del recorrido de las rutas elegidas

\begin{tabular}{|c|l|c|c|c|}
\hline Cód. & Rutas & $\begin{array}{c}\text { Duración } \\
\text { ruta (min) }\end{array}$ & $\begin{array}{c}\text { Demanda } \\
\text { de } \\
\text { personas }\end{array}$ & Descanso \\
\hline 107 & $\begin{array}{l}\text { Pablo Bello } \\
\text { Cedritos }\end{array}$ & 56 & 5182 & 10 \\
\hline 103 & $\begin{array}{l}\text { Maíz } \\
\text { Amarillo }\end{array}$ & 53 & 4145 & 20 \\
\hline 100 & Llano Largo & 64 & 4182 & 10 \\
\hline
\end{tabular}

Como se mencionó en la metodología, para completar los datos de entrada necesarios solo hace falta el análogo al Kanban, que es la Capacidad Disponible promedio de los buses, la cual es calculada como un promedio de la capacidad del total de todos los buses útiles en Fusagasugá asociados al Sistema Integrado de Transporte: la mayoría de los carros son Nissan Urban o similares, modelo 1997 con algunas excepciones de nuevos modelos NKR de mayor capacidad, sin embargo es un cambio progresivo de tecnología que demanda el municipio. Para efectos de facilidad en el proceso del algoritmo se estandarizan los datos con las suposiciones anteriormente descritas (Tabla 4). Por último, el índice " 1 " que se explica con la ecuación 1.2, se establece bajo la suposición de que la flota de buses se estandariza en su capacidad a 16 pasajeros y que, aunque hay diferencias entre los modelos, éstos se compensan unos a otros y el ponderado de capacidad se mantiene. Por ende la ecuación se vuelve 1 , y así para todos los buses.

\begin{tabular}{|c|c|c|c|}
\hline \multicolumn{4}{|c|}{ Modelos de los buses en general } \\
\hline Empresa & $\begin{array}{l}\text { Número } \\
\text { de buses }\end{array}$ & $\begin{array}{c}\text { Capacidad } \\
\text { por } \\
\text { modelos }\end{array}$ & $\begin{array}{c}\text { Capacidad } \\
\text { total }\end{array}$ \\
\hline $\begin{array}{l}\text { Modelo } 2010 \\
\text { en adelante }\end{array}$ & 26 & 19 & 494 \\
\hline $\begin{array}{l}\text { Modelos } 2010 \\
\text { al } 99\end{array}$ & 125 & 16 & 2000 \\
\hline $\begin{array}{l}\text { Modelos } 98 \text { o } \\
\text { inferiores }\end{array}$ & 110 & 15 & 1650 \\
\hline & & Promedio & 15,88 \\
\hline
\end{tabular}

Por último, es necesario tener en cuenta los horarios de salida de cada ruta, las cuales son las mismas para cada día de la semana. Estos son determinados por la empresa. Como cada ruta presenta recorridos diferentes por zonas diferentes, los tiempos de inicio de operación por ruta dependen, en la mayoría de los casos, de puntos clave que arrancan operación a horas similares o que es necesario que los buses transporten personas a estos lugares a horas tempranas. Por estos factores las empresas han determinado los siguientes horarios de salida para cada ruta (Tabla 5).

Tabla 5. Datos de inicio de jornada laboral y de cantidad diarias de horas disponibles para trabajar por ruta:

\begin{tabular}{|c|c|c|c|c|c|}
\hline \multicolumn{2}{|c|}{107} & \multicolumn{2}{c|}{103} & \multicolumn{2}{c|}{100} \\
\hline $\begin{array}{c}\text { Días } \\
\text { semana }\end{array}$ & $\begin{array}{c}\text { Horas } \\
\text { día }\end{array}$ & $\begin{array}{c}\text { Días } \\
\text { semana }\end{array}$ & $\begin{array}{c}\text { Horas } \\
\text { día }\end{array}$ & $\begin{array}{c}\text { Días } \\
\text { semana }\end{array}$ & $\begin{array}{c}\text { Horas } \\
\text { día }\end{array}$ \\
\hline 7 & 17 & 7 & 16,667 & 7 & 17 \\
\hline $\begin{array}{c}\text { Empieza } \\
\text { jornada }\end{array}$ & 5,333 & $\begin{array}{c}\text { Empieza } \\
\text { jornada }\end{array}$ & 5,333 & $\begin{array}{c}\text { Empieza } \\
\text { jornada }\end{array}$ & 5 \\
\hline Descanso & 10 & Descanso & 20 & Descanso & 10 \\
\hline
\end{tabular}

Se puede tener en cuenta que rutas como la 107 y 103 comienzan su hora de operación similarmente, pues ellos van desde sus paraderos que quedan en zonas residenciales y pasan a través de lugares claves como el centro o los colegios y se hace indispensable el arribo a tiempo, de comerciantes, docentes y estudiantes a realizar sus labores del día a día. En contraste, rutas dirigidas por ejemplo al Terminal de Transportes de Fusagasugá tienen en cuenta que solo tienen operaciones entre $7 \mathrm{am}$ y $7 \mathrm{pm}$, por ende el horario en inicio de operación es más atrasado y la cantidad de horas disponibles para trabajar más limitadas.

\section{Generar la frecuencia de surtida}

Para generar la frecuencia de surtida se aplica la ecuación 4.1 para obtener la demanda real, cual es la verdadera demanda que el vehículo puede soportar proporcionalmente en comparación al resto de los buses inscritos en dicha ruta. De esta forma se pueden generar horarios de salida para cada día ya estipulados conforme a la capacidad real de los vehículos. Sin embargo para este caso de aplicación se estandariza que el Sistema Integrado de Transporte tiene un numero de buses $\mathrm{j}$ con una capacidad estándar igual entre todas; de esta manera los horarios de salida son los mismos y se utilizan todos los días del año o hasta que sea necesario un nuevo ajuste. 
Posteriormente se tabula la ecuación 4.2 para obtener la cantidad de recorridos que debería hacer cada carro y la frecuencia de surtida (ecuación 4.3) que indica cómo se va a intercalar la salida de los buses (Tabla 6).

Tabla 6. Frecuencias de surtida para los primeros 4 buses de la ruta 107 Pablo Bello Cedritos. El resultado de aplicar las ecuaciones 4.1, 4.2 y 4.3 tabuladas en Excel reflejan un distanciamiento de 3,15 minutos entre cada uno de los componentes.

\begin{tabular}{|c|c|c|c|}
\hline Bus $\mathbf{j}$ & $\begin{array}{c}\text { Demanda } \\
\text { Real }\end{array}$ & $\begin{array}{c}\text { Kanban } \\
\text { requerido }\end{array}$ & $\begin{array}{c}\text { Frecuencia de } \\
\text { surtida }\end{array}$ \\
\hline B1 & 5182 & 323,9 & 3,15 \\
\hline B2 & 5182 & 323,9 & 3,15 \\
\hline B3 & 5182 & 323,9 & 3,15 \\
\hline B4 & 5182 & 323,9 & 3,15 \\
\hline
\end{tabular}

La diferencia que existe con el algoritmo de aproximación de surtida de materiales es que para este caso esta frecuencia no será el tiempo de surtida del material $j$, sino que será el distanciamiento entre salidas de cada uno de los buses j. Este cambio se evidencia observando la Tabla 7.

Para la ruta 107 arroja una frecuencia de salida de 3,15 minutos, mientras que para la 103 arroja una de 3,94 minutos (Lo que tiene sentido entre la cantidad de demanda y de buses para cada ruta).
El valor de la frecuencia es determinante en el momento de generar los horarios de salida de cada uno de los buses.

\section{Bosquejo del horario de salida: Raíz HSj}

Con las frecuencias de salida asignadas a cada ruta, la raíz HSj tiene en cuenta el distanciamiento de los buses, el tiempo de recorrido de cada ruta y además un tiempo de descanso para cada conductor del bus (Ecuación 5.1) (Tabla 7). Es así que se tabulan los horarios de salida de cada bus teniendo en cuenta la restricción de horarios de trabajo permitidos para cada ruta.

\section{Horarios de salida}

Con la raíz HSj generada se obtienen los horarios de salida de los vehículos en hora y minuto (Reloj) que deben cumplir los buses en el día. Como existen buses que empiezan su jornada más temprano que otros, y algunos realizan más recorridos que otros, se propone no alterar los horarios de salida (y así mantenerlos estandarizados) sino que mejor se roten los vehículos cada vez que tengan que utilizar dicha ruta: si a un bus j se le asignó el horario del bus B1 de la ruta 107, la próxima vez que tenga la ruta 107 rotará su lugar a ser el bus B2, y así se conserva la igualdad en los horarios. Ver Tabla 8.

\begin{tabular}{|c|c|c|c|c|c|c|c|c|c|c|c|c|c|c|c|c|c|c|}
\hline \multicolumn{19}{|c|}{107 PABLO BELLO CEDRITOS } \\
\hline \multicolumn{19}{|c|}{1020} \\
\hline B1 & B2 & B3 & B4 & B5 & B6 & B7 & B8 & B9 & B10 & B11 & B12 & B13 & B14 & B15 & B16 & B17 & B18 & B19 \\
\hline 0,0 & 3,1 & 6,3 & 9,4 & 12,6 & 15,7 & 18,9 & 22,0 & 25,2 & 28,3 & 31,5 & 34,6 & 37,8 & 40,9 & 44,1 & 47,2 & 50,4 & 53,5 & 56,7 \\
\hline 66,0 & 69,1 & 72,3 & 75,4 & 78,6 & 81,7 & 84,9 & 88,0 & 91,2 & 94,3 & 97,5 & 100,6 & 103,8 & 106,9 & 110,1 & 113,2 & 116,4 & 119,5 & 122,7 \\
\hline 132,0 & 135,1 & 138,3 & 141,4 & 144,6 & 147,7 & 150,9 & 154,0 & 157,2 & 160,3 & 163,5 & 166,6 & 169,8 & 172,9 & 176,1 & 179,2 & 182,4 & 185,5 & 188,7 \\
\hline 198,0 & 201,1 & 204,3 & 207,4 & 210,6 & 213,7 & 216,9 & 220,0 & 223,2 & 226,3 & 229,5 & 232,6 & 235,8 & 238,9 & 242,1 & 245,2 & 248,4 & 251,5 & 254,7 \\
\hline 264,0 & 267,1 & 270,3 & 273,4 & 276,6 & 279,7 & 282,9 & 286,0 & 289,2 & 292,3 & 295,5 & 298,6 & 301,8 & 304,9 & 308,1 & 311,2 & 314,4 & 317,5 & 320,7 \\
\hline 330,0 & 333,1 & 336,3 & 339,4 & 342,6 & 345,7 & 348,9 & 352,0 & 355,2 & 358,3 & 361,5 & 364,6 & 367,8 & 370,9 & 374,1 & 377,2 & 380,4 & 383,5 & 386,7 \\
\hline 396,0 & 399,1 & 402,3 & 405,4 & 408,6 & 411,7 & 414,9 & 418,0 & 421,2 & 424,3 & 427,5 & 430,6 & 433,8 & 436,9 & 440,1 & 443,2 & 446,4 & 449,5 & 452,7 \\
\hline 462,0 & 465,1 & 468,3 & 471,4 & 474,6 & 477,7 & 480,9 & 484,0 & 487,2 & 490,3 & 493,5 & 496,6 & 499,8 & 502,9 & 506,1 & 509,2 & 512,4 & 515,5 & 518,7 \\
\hline 528,0 & 531,1 & 534,3 & 537,4 & 540,6 & 543,7 & 546,9 & 550,0 & 553,2 & 556,3 & 559,5 & 562,6 & 565,8 & 568,9 & 572,1 & 575,2 & 578,4 & 581,5 & 584,7 \\
\hline 594,0 & 597,1 & 600,3 & 603,4 & 606,6 & 609,7 & 612,9 & 616,0 & 619,2 & 622,3 & 625,5 & 628,6 & 631,8 & 634,9 & 638,1 & 641,2 & 644,4 & 647,5 & 650,7 \\
\hline 660,0 & 663,1 & 666,3 & 669,4 & 672,6 & 675,7 & 678,9 & 682,0 & 685,2 & 688,3 & 691,5 & 694,6 & 697,8 & 700,9 & 704,1 & 707,2 & 710,4 & 713,5 & 716,7 \\
\hline 726,0 & 729,1 & 732,3 & 735,4 & 738,6 & 741,7 & 744,9 & 748,0 & 751,2 & 754,3 & 757,5 & 760,6 & 763,8 & 766,9 & 770,1 & 773,2 & 776,4 & 779,5 & 782,7 \\
\hline 792,0 & \begin{tabular}{|l}
795,1 \\
\end{tabular} & 798,3 & 801,4 & 804,6 & \begin{tabular}{|l}
807,7 \\
\end{tabular} & 810,9 & 814,0 & 817,2 & 820,3 & 823,5 & 826,6 & 829,8 & 832,9 & 836,1 & 839,2 & 842,4 & 845,5 & 848,7 \\
\hline 858,0 & 861,1 & 864,3 & 867,4 & 870,6 & 873,7 & 876,9 & 880,0 & 883,2 & 886,3 & 889,5 & 892,6 & 895,8 & 898,9 & 902,1 & 905,2 & 908,4 & 911,5 & 914,7 \\
\hline 924,0 & 927,1 & 930,3 & 933,4 & 936,6 & 939,7 & 942,9 & 946,0 & 949,2 & 952,3 & 955,5 & 958,6 & 961,8 & 964,9 & 968,1 & 971,2 & 974,4 & 977,5 & 980,7 \\
\hline 990,0 & 993,1 & 996,3 & 999,4 & 1002,6 & 1005,7 & 1008,9 & 1012,0 & 1015,2 & 1018,3 & & & & & & & & & \\
\hline
\end{tabular}




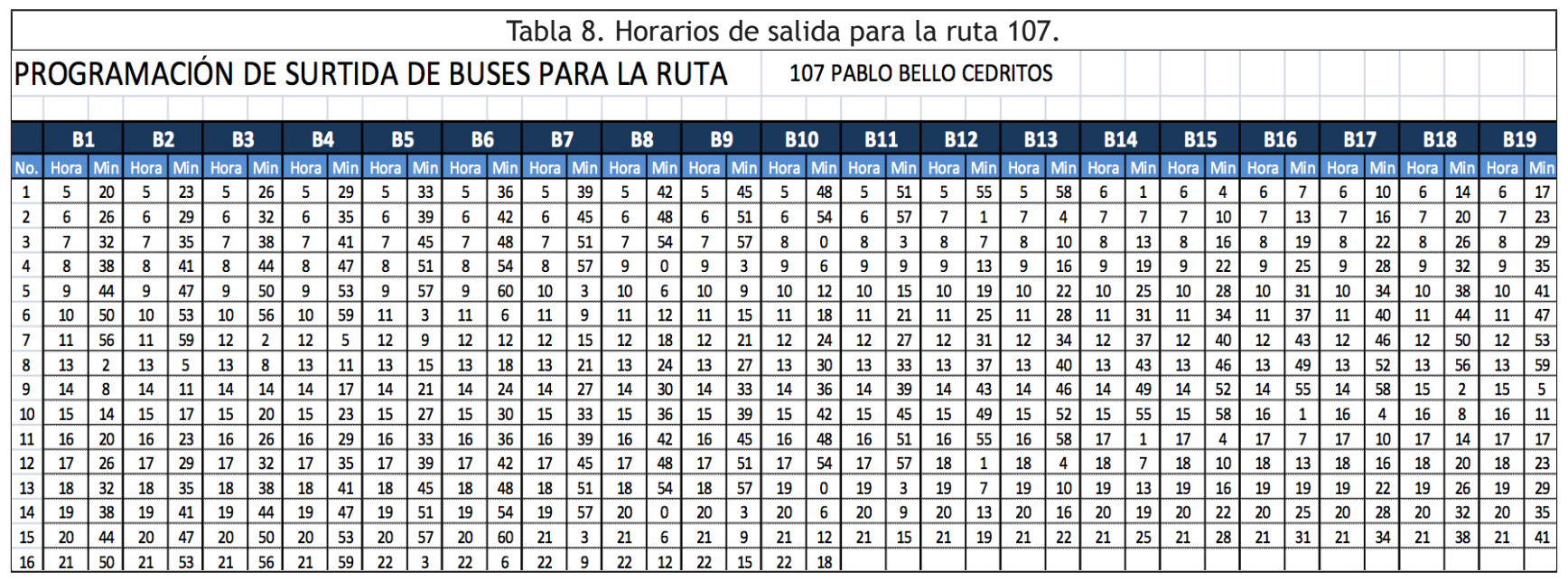

\begin{tabular}{|c|c|c|c|c|c|c|c|c|c|c|c|c|c|c|c|c|c|c|c|c|c|c|c|c|c|c|c|c|c|c|c|c|c|c|c|c|c|c|}
\hline & & & & & & & & & & & & & & Hor & a & & . & sali & da & par & a la & $\mathrm{ru}$ & ta 1 & 103 & & & & & & & & & & & & & & \\
\hline & 20 & $\mathrm{GR}$ & AM & $A C$ & IÓ & V & E S & UP & TTID & DA & DE & $B L$ & JSES & $S P$ & AR & A L & A & $R U$ & TA & & & $3 \mathrm{M}$ & AIZ A & $\mathrm{AM}$ & ARILL & & & & & & & & & & & & & \\
\hline & B & 1 & B & & B & & B & 4 & B & & B & & B7 & & B & & B & & B1 & & B1 & & B1 & 12 & B1: & & B1 & 14 & B1 & 15 & B1 & 16 & B1 & & B1 & & B1 & \\
\hline & tora & Mir & Hora & Min & Hora & Min & Hora & Min & Hora & Min & Hora & $\mathrm{Min}$ & Hora & Min & Hora & Min & Hora & Min & Hora & Min & Hora & Min & Hora & Min & & & Hora & & Hora & Min & Hora & & & Min & & Min & & Min \\
\hline 1 & 5 & 20 & 5 & \begin{tabular}{|l|}
24 \\
\end{tabular} & 5 & 28 & 5 & \begin{tabular}{|l|}
32 \\
\end{tabular} & 5 & 36 & 5 & 40 & \begin{tabular}{|l|}
5 \\
\end{tabular} & 44 & 5 & 48 & 5 & \begin{tabular}{|l|}
51 \\
\end{tabular} & 5 & 55 & \begin{tabular}{|l|}
5 \\
\end{tabular} & \begin{tabular}{|l|}
59 \\
\end{tabular} & \begin{tabular}{|l|}
6 \\
\end{tabular} & 3 & 6 & 7 & 6 & \begin{tabular}{|c|}
11 \\
\end{tabular} & 6 & \begin{tabular}{|c|}
15 \\
\end{tabular} & \begin{tabular}{|l|}
6 \\
\end{tabular} & \begin{tabular}{|l|}
19 \\
\end{tabular} & \begin{tabular}{|l|}
6 \\
\end{tabular} & 23 & \begin{tabular}{|l|}
6 \\
\end{tabular} & \begin{tabular}{|l|}
27 \\
\end{tabular} & \begin{tabular}{|l|}
6 \\
\end{tabular} & 31 \\
\hline 2 & 6 & 33 & 6 & 37 & 6 & 41 & 6 & 45 & 6 & 49 & 6 & 53 & 6 & 57 & 7 & 1 & 7 & 4 & 7 & 8 & 7 & 12 & 7 & 16 & 7 & 20 & 7 & 24 & 7 & 28 & 7 & 32 & 7 & 36 & 7 & 40 & 7 & 44 \\
\hline 3 & 7 & 46 & 7 & 50 & 7 & 54 & 7 & 58 & 8 & 2 & 8 & 6 & 8 & 10 & 8 & 14 & 8 & 17 & 8 & 21 & 8 & 25 & 8 & 29 & 8 & 33 & 8 & 37 & 8 & 41 & 8 & 45 & 8 & \begin{tabular}{|l|l|}
49 \\
\end{tabular} & 8 & 53 & 8 & 57 \\
\hline 4 & 8 & 59 & 9 & 3 & 9 & 7 & 9 & 11 & 9 & 15 & 9 & 19 & 9 & 23 & 9 & 27 & 9 & 30 & 9 & 34 & 9 & 38 & 9 & 42 & 9 & 46 & 9 & 50 & 9 & 54 & 9 & 58 & 10 & 2 & 10 & 6 & 10 & 10 \\
\hline 5 & 10 & 12 & 10 & 16 & 10 & 20 & 10 & 24 & 10 & 28 & 10 & 32 & 10 & 36 & 10 & 40 & 10 & 43 & 10 & 47 & 10 & 51 & 10 & 55 & 10 & 59 & 11 & 3 & 11 & \begin{tabular}{|l|} 
\\
\end{tabular} & 11 & 11 & 11 & 15 & 11 & 19 & 11 & 23 \\
\hline 6 & 11 & 25 & 11 & 29 & 11 & 33 & 11 & 37 & 11 & 41 & 11 & 45 & 11 & 49 & 11 & 53 & 11 & 56 & 12 & 0 & \begin{tabular}{|l|}
12 \\
\end{tabular} & 4 & 12 & 8 & 12 & 12 & 12 & 16 & 12 & 20 & 12 & 24 & 12 & 28 & 12 & 32 & 12 & 36 \\
\hline 7 & 12 & 38 & 12 & 42 & 12 & 46 & 12 & 50 & 12 & 54 & 12 & 58 & 13 & 2 & 13 & 6 & 13 & 9 & 13 & 13 & \begin{tabular}{|l|}
13 \\
\end{tabular} & 17 & 13 & 21 & 13 & 25 & 13 & 29 & 13 & 33 & 13 & 37 & 13 & \begin{tabular}{|l|}
4 \\
1
\end{tabular} & 13 & 45 & 13 & 49 \\
\hline 8 & 13 & 51 & 13 & 55 & 13 & 59 & 14 & 3 & 14 & 7 & 14 & 11 & 14 & 15 & 14 & 19 & 14 & 22 & 14 & 26 & 14 & 30 & 14 & 34 & 14 & 38 & 14 & 42 & 14 & 46 & \begin{tabular}{|l|l|}
14 \\
\end{tabular} & 50 & 14 & 54 & 14 & 58 & 15 & 2 \\
\hline 9 & 15 & 4 & 15 & 8 & 15 & 12 & 15 & 16 & 15 & 20 & 15 & 24 & 15 & 28 & 15 & 32 & 15 & 35 & 15 & \begin{tabular}{|l|}
39 \\
\end{tabular} & 15 & 43 & 15 & 47 & 15 & 51 & 15 & 55 & 15 & 59 & 16 & 3 & 16 & \begin{tabular}{|l|}
7 \\
\end{tabular} & 16 & 11 & 16 & 15 \\
\hline 10 & 16 & 17 & 16 & 21 & 16 & 25 & 16 & 29 & 16 & 33 & 16 & 37 & 16 & 41 & 16 & 45 & 16 & 48 & 16 & 52 & \begin{tabular}{|l|}
16 \\
\end{tabular} & 56 & 17 & 0 & 17 & 4 & 17 & 8 & 17 & 12 & 17 & 16 & 17 & 20 & 17 & 24 & 17 & 28 \\
\hline 11 & 17 & 30 & 17 & 34 & 17 & 38 & 17 & 42 & 17 & 46 & 17 & 50 & 17 & 54 & 17 & 58 & 18 & 1 & 18 & 5 & \begin{tabular}{|l|}
18 \\
\end{tabular} & 9 & 18 & 13 & 18 & 17 & 18 & 21 & 18 & 25 & 18 & 29 & 18 & 33 & 18 & 37 & 18 & 41 \\
\hline 12 & 18 & 43 & 18 & 47 & 18 & 51 & 18 & 55 & 18 & 59 & 19 & 3 & 19 & 7 & 19 & 11 & 19 & 14 & 19 & 18 & \begin{tabular}{|l|}
19 \\
\end{tabular} & 22 & 19 & 26 & 19 & 30 & 19 & 34 & 19 & 38 & 19 & 42 & 19 & 46 & 19 & 50 & 19 & 54 \\
\hline 13 & 19 & 56 & 19 & 60 & 20 & 4 & 20 & 8 & 20 & 12 & 20 & 16 & 20 & 20 & 20 & 24 & 20 & 27 & 20 & 31 & 20 & 35 & 20 & 39 & 20 & 43 & 20 & 47 & 20 & 51 & 20 & 55 & 20 & \begin{tabular}{|l|}
59 \\
\end{tabular} & 21 & 3 & 21 & \\
\hline 14 & 21 & 9 & 21 & 13 & 21 & 17 & 21 & 21 & 21 & 25 & 21 & 29 & \begin{tabular}{|l|}
21 \\
\end{tabular} & 33 & 21 & 37 & \begin{tabular}{|l|}
21 \\
\end{tabular} & \begin{tabular}{|l|}
40 \\
\end{tabular} & 21 & \begin{tabular}{|l|}
44 \\
\end{tabular} & 21 & \begin{tabular}{|l|} 
\\
\end{tabular} & \begin{tabular}{|l|}
21 \\
\end{tabular} & 52 & 21 & 56 & & & & & & & & & & & & \\
\hline
\end{tabular}

\begin{tabular}{|c|c|c|c|c|c|c|c|c|c|c|c|c|c|c|c|c|c|c|c|c|c|c|c|c|c|c|c|c|c|c|c|c|c|c|c|c|c|c|c|c|}
\hline \multicolumn{41}{|c|}{ Horarios de salida para la ruta 100.} \\
\hline \multirow{3}{*}{\multicolumn{2}{|c|}{$\begin{array}{ll}\text { PROGR } \\
\text { D1 }\end{array}$}} & $R A$ & $\mathrm{MAC}$ & CIÓ & N [ & ES & ALID, & $\mathrm{ADE}$ & $\mathrm{BU}$ & JSES & 5 PAF & RA LA & $A R U$ & UTA & & 100 & LLANC & IO LA & & & & & & & & & & & & & & & & & & & & & & \\
\hline & & & & 02 & & D3 & & D4 & & D5 & & D6 & & D7 & D8 & 88 & DS & 9 & D1 & & D1 & 11 & D1: & 12 & D1 & 13 & & 014 & D15 & & D1 & 16 & & 017 & & 018 & D19 & & D2 & \\
\hline & & & Hora & & Hore & & \begin{tabular}{|l|l|}
$n$ & Hor \\
\end{tabular} & & Hora & Min & \begin{tabular}{|l|l|} 
Hor \\
\end{tabular} & a Min & Hora & Min & Hora & Min & & Min & & & Hora & Min & Hora & Min & Hora & & & Min & Hora & & & Min & & Min & & Min & & Min & & \\
\hline & & 0 & 5 & & 5 & 8 & & & 5 & 16 & \begin{tabular}{|l|l}
5 & 5 \\
\end{tabular} & 20 & \begin{tabular}{|l|}
5 \\
\end{tabular} & \begin{tabular}{|l|}
23 \\
\end{tabular} & \begin{tabular}{|l|}
5 \\
\end{tabular} & \begin{tabular}{|l|}
27 \\
\end{tabular} & \begin{tabular}{|l|}
5 \\
\end{tabular} & & \begin{tabular}{l|l}
5 \\
\end{tabular} & 35 & \begin{tabular}{|l|}
5 \\
\end{tabular} & \begin{tabular}{|l|}
39 \\
\end{tabular} & \begin{tabular}{|l|}
5 \\
\end{tabular} & \begin{tabular}{|l|}
43 \\
\end{tabular} & \begin{tabular}{|l|l|} 
\\
\end{tabular} & \begin{tabular}{|l|}
47 \\
\end{tabular} & \begin{tabular}{|l|} 
\\
\end{tabular} & \begin{tabular}{|l|}
51 \\
\end{tabular} & \begin{tabular}{|l|l}
5 &
\end{tabular} & 55 & \begin{tabular}{|c|}
5 \\
\end{tabular} & \begin{tabular}{|l|}
59 \\
\end{tabular} & 6 & 2 & 6 & 6 & 6 & 10 & 6 & 14 \\
\hline & & 14 & 6 & 18 & 6 & 22 & 6 & 26 & 6 & 30 & 6 & 34 & 6 & 37 & 6 & 41 & 6 & 45 & 6 & 49 & 6 & 53 & 6 & 57 & 7 & 1 & 7 & 5 & 7 & 9 & 7 & 13 & 7 & 16 & 7 & 20 & 7 & 24 & 7 & \\
\hline 3 & & 28 & 7 & 32 & 7 & 36 & 7 & 40 & 7 & 44 & 7 & 48 & 7 & 51 & 7 & 55 & 7 & 59 & 8 & 3 & 8 & 7 & 8 & 11 & 8 & 15 & 8 & 19 & 8 & 23 & 8 & 27 & 8 & 30 & 8 & 34 & 8 & 38 & 8 & \\
\hline & 8 & 42 & 8 & 46 & 8 & 50 & 8 & 54 & 8 & 58 & 9 & 2 & 9 & 5 & 9 & 9 & 9 & 13 & 9 & 17 & 9 & 21 & 9 & 25 & 9 & 29 & 9 & 33 & 9 & 37 & 9 & 41 & 9 & 44 & 9 & 48 & 9 & 52 & 9 & \\
\hline & & 56 & 9 & 60 & 10 & 4 & 10 & 8 & 10 & 12 & 10 & 16 & 10 & 19 & 10 & 23 & 10 & 27 & 10 & 31 & 10 & 35 & 10 & 39 & 10 & 43 & 10 & 47 & 10 & 51 & 10 & 55 & 10 & 58 & 11 & 2 & & 6 & 11 & \\
\hline & & 10 & 11 & 14 & 11 & 18 & 11 & 22 & 11 & 26 & 11 & 30 & 11 & 33 & 11 & 37 & 11 & 41 & & 45 & 11 & 49 & 11 & 53 & 11 & 57 & 12 & 1 & 12 & 5 & 12 & 9 & 12 & 12 & 12 & 16 & & 20 & 12 & \\
\hline & & 24 & 12 & 28 & 12 & 32 & 12 & 36 & 12 & 40 & 12 & 44 & 12 & 47 & & 51 & 12 & 55 & & 59 & 13 & 3 & 13 & 7 & 13 & 11 & 13 & 15 & 13 & 19 & 13 & 23 & 13 & 26 & 13 & 30 & & 34 & 13 & \\
\hline & & 38 & 13 & 42 & 13 & 46 & 13 & 50 & 13 & 54 & 13 & 58 & 14 & & 14 & 5 & 14 & 9 & & 13 & 14 & 17 & 14 & 21 & 14 & 25 & 14 & 29 & 14 & & 14 & 37 & 14 & 40 & 14 & 44 & & & 14 & \\
\hline & 14 & 52 & 14 & 56 & 14 & 60 & 15 & 4 & 15 & 8 & 15 & 12 & 15 & 15 & 15 & 19 & 15 & 23 & 15 & 27 & 15 & 31 & 15 & 35 & 15 & 39 & 15 & 43 & 15 & 47 & 15 & 51 & 15 & 54 & 15 & 58 & 16 & & 16 & \\
\hline & 16 & 6 & 16 & 10 & 16 & 14 & 16 & 18 & 16 & 22 & 16 & 26 & 16 & 29 & 16 & 33 & 16 & 37 & 16 & 41 & 16 & 45 & 16 & 49 & 16 & 53 & 16 & 57 & 17 & & 17 & 5 & 17 & 8 & 17 & 12 & 17 & 16 & 17 & 20 \\
\hline & & 20 & 17 & 24 & 17 & 28 & 17 & 32 & 17 & 36 & 17 & 40 & 17 & 43 & 17 & 47 & 17 & 51 & 17 & 55 & 17 & 59 & 18 & & 18 & & 18 & & 18 & & 18 & & 18 & & 18 & & & & 18 & 20 \\
\hline & & 34 & 18 & 38 & 18 & 42 & 18 & 46 & 18 & 50 & 18 & 54 & 18 & 57 & 19 & 1 & 19 & 5 & 19 & 9 & 19 & 13 & 19 & 17 & 19 & 21 & 19 & 25 & 19 & 29 & 19 & 33 & 19 & 36 & 19 & 40 & 19 & 44 & 19 & 48 \\
\hline & 19 & 48 & 19 & \begin{tabular}{|l}
52 \\
\end{tabular} & 19 & 56 & $\begin{array}{ll}19 \\
\end{array}$ & $\begin{array}{ll}9 & 60 \\
& \end{array}$ & 20 & 4 & 20 & 8 & 20 & $\mid 11$ & 20 & \begin{tabular}{|l|}
15 \\
\end{tabular} & \begin{tabular}{|l|}
20 \\
21
\end{tabular} & \begin{tabular}{|l|}
19 \\
323
\end{tabular} & 20 & 23 & 20 & 27 & 20 & 31 & 20 & 35 & 20 & 39 & 20 & & 20 & 47 & 20 & 50 & 20 & 54 & \begin{tabular}{|l|l|l}
20 & \\
\end{tabular} & 58 & 21 & 2 \\
\hline
\end{tabular}

\section{CONCLUSIONES}

Los sistemas de transporte público, en muchos casos, se han caracterizado por su ineficiencia derivada de una mala planeación, organización y control de las funciones que desempeñan. Una de las más notorias deficiencias es el inconformismo generado por un servicio que debería ser constante, en cuando a frecuencias de rutas se refiere, pero que por el contrario, presenta un alto grado de variación.
Un sistema de transporte público es vital para cualquier ciudad, ya que permite la movilidad de sus habitantes dentro de ésta en tiempos cortos. Por esta razón, para que dicho sistema pueda satisfacer en gran parte las demandas de sus clientes- los pasajeros-, se ha de tener un orden estricto de salida - horario- con el fin de que las frecuencias de los buses sean constantes. No importa el método por el que se diseñe este orden: Programación Lineal Entera (PLE), métodos matemáticos del "Problema 
del Transporte" o como la alternativa que se presenta en este artículo, una analogía del algoritmo de aproximación en manufactura esbelta. Ya que si se tienen en cuenta las bases de esta filosofía se pueden encontrar excelentes soluciones logísticas de optimización.

Por todo lo anterior, al tener un orden de salida de buses para las diferentes rutas que conforman un sistema de transporte público se mejorara significativamente la percepción de servicio. Con un sistema de transporte público que sea ordenado y en el cual las frecuencias de salida estén diseñadas para suplir las necesidades de los usuarios, todos los actores de un entorno ciudadano se ven beneficiados sustancialmente. El algoritmo de aproximación propuesto es una técnica novedosa que permite programar las rutas de buses urbanos, que a diferencia de las técnicas clásicas de optimización, es computacionalmente más eficiente.

Con el algoritmo de aproximación se logran programar rutas urbanas de acuerdo con la capacidad de la ruta y el tipo de bus, entregando como resultado el programa operativo de despacho. El algoritmo es una técnica de carácter operativo para las compañías de transporte público que permite en menor tiempo, obtener programaciones de acuerdo con el flujo de pasajeros.

Cabe resaltar que el algoritmo es una base que puede dejar en el aire factores del transporte urbano que influyen directamente en la calidad del servicio, pero como tal, el caso de aplicación se ejecutó en un municipio donde se pudo realizar una adecuada limitación del sistema de transporte urbano en estudio.

\section{REFERENCIAS BIBLIOGRÁFICAS}

1. Universidad Pedagógica y Tecnológica de Colombia, "Sistemas de Transporte en TunjaCaracterísticas». Grupo de Investigación y
Desarrollo en Planeación y Operación del Transporte - GIDPOT, Tunja, 2012.

2. J. d. D. Ortazar, Modelos del Transporte, SantanderEspaña, Universidad de Cantabria, 2011.

3. M. Urrutia, Evaluacion del Sistema de Transporte Público en Bogotá. Bogotá: Fundación para la Educación Superior y el Desarrollo, 1981.

4. A. y. M. d. I. S. Ferrer, Un modelo de transporte de distribución, Madrid: Actas del XIV Congreso de Ingeniería Mecánica, 2000.

5. Y. Monden, El Sistema de Producción de Toyota, Ediciones Macchi, 1990.

6. L. Cuatrecasas, Claves del Lean Management: un enfoque para la alta competitividad en un mundo globalizado, Barcelona: Editorial Gestión 2000, 2006.

7. M. Rajadell Carreras y J. L. Sánchez García, Lean Manufacturing: La evidencia de una necesidad, Madrid: Ediciones Díaz de Santos, 2010.

8. J. Womack, Lean Thinking: cómo utilizar el pensamiento Lean para eliminar los despilfarros y crear valor en la empresa, Barcelona: Editorial Gestión 2000, 2004.

9. G. Lieberman y H. Frederick, Investigación de Operaciones, México: Editorial McGraw-Hill, 2002.

10. T. H. A., Investigación de Operaciones, Pearson Educación, 2004.

11. J. a. I. Barreras y J. E. O. Tiznado, Modelo matemático de transporte aplicado a una compañía, Universidad Autónoma de Baja California, México, 2011.

12. Abarca, «Abarca Ecodiseño,» 2012. [En línea]. Available: http://www.abarcaeco.com/presentacion/ ventajas-del-ecodiseno. [Último acceso: 2311 2012]. 